\title{
Non-existence of bipartite graphs of diameter at least 4 and defect 2
}

\author{
Guillermo Pineda-Villavicencio
}

Received: 3 January 2010 / Accepted: 8 November 2010 / Published online: 20 November 2010

(C) Springer Science+Business Media, LLC 2010

\begin{abstract}
The Moore bipartite bound represents an upper bound on the order of a bipartite graph of maximum degree $\Delta$ and diameter $D$. Bipartite graphs of maximum degree $\Delta$, diameter $D$ and order equal to the Moore bipartite bound are called Moore bipartite graphs. Such bipartite graphs exist only if $D=2,3,4$ and 6 , and for $D=$ $3,4,6$, they have been constructed only for those values of $\Delta$ such that $\Delta-1$ is a prime power.
\end{abstract}

The scarcity of Moore bipartite graphs, together with the applications of such large topologies in the design of interconnection networks, prompted us to investigate what happens when the order of bipartite graphs misses the Moore bipartite bound by a small number of vertices. In this direction the first class of graphs to be studied is naturally the class of bipartite graphs of maximum degree $\Delta$, diameter $D$, and two vertices less than the Moore bipartite bound (defect 2), that is, bipartite $(\Delta, D,-2)$ graphs.

For $\Delta \geq 3$ bipartite $(\Delta, 2,-2)$-graphs are the complete bipartite graphs with partite sets of orders $\Delta$ and $\Delta-2$. In this paper we consider bipartite $(\Delta, D,-2)$ graphs for $\Delta \geq 3$ and $D \geq 3$. Some necessary conditions for the existence of bipartite $(\Delta, 3,-2)$-graphs for $\Delta \geq 3$ are already known, as well as the non-existence of bipartite $(\Delta, D,-2)$-graphs with $\Delta \geq 3$ and $D=4,5,6,8$. Furthermore, it had been conjectured that bipartite $(\Delta, D,-2)$-graphs for $\Delta \geq 3$ and $D \geq 4$ do not exist. Here, using graph spectra techniques, we completely settle this conjecture by proving the non-existence of bipartite $(\Delta, D,-2)$-graphs for all $\Delta \geq 3$ and all $D \geq 6$.

Keywords Degree/diameter problem - Moore bipartite bound · Moore bipartite graphs, defect · Dickson polynomials of the second kind

G. Pineda-Villavicencio $(\bowtie)$

Centre for Informatics and Applied Optimization, University of Ballarat, Mount Helen,

Victoria 3353, Australia

e-mail: work@guillermo.com.au 


\section{Introduction}

The ever increasing need for the design of interconnection networks has motivated the study of large graphs of given maximum degree and diameter. While the modelling of a network by such graphs does not take into account implementation factors of the network, it does provide an effective means of abstraction to study many relevant network properties $[6,9,12,19,20]$.

Since the features of an interconnection network depend considerably on the particular application, it is then understandable that many interpretations about the "optimality" of a network coexist. One possible interpretation is presented as follows; see [9, p. 18], [12, p. 168], and [19, p. 91].

An optimal network contains the maximum possible number of nodes, given a limit on the number of connections attached to a node and a limit on the diameter of the network.

This interpretation is encapsulated by the so-called degree/diameter problem [15], which can be stated as follows.

Degree/diameter problem: Given natural numbers $\Delta \geq 2$ and $D \geq 2$, find the largest possible number $N_{\Delta, D}$ of vertices in a graph of maximum degree $\Delta$ and diameter $D$.

It is known that the Moore bound $M_{\Delta, D}$, defined below, provides an upper bound for $N_{\Delta, D}$. Regular graphs of degree $\Delta$, diameter $D$ and order $M_{\Delta, D}$ are called Moore graphs. Non-trivial Moore graphs (that is, those with $\Delta \geq 3$ and $D \geq 2$ ) exist only for diameter 2 , in which case their degree is $2,3,7$ or possibly 57 ; see [15].

$$
M_{\Delta, D}= \begin{cases}1+\Delta \frac{(\Delta-1)^{D}-1}{\Delta-2} & \text { if } \Delta>2 \\ 2 D+1 & \text { if } \Delta=2\end{cases}
$$

In the design of interconnection networks with bidirectional communication channels, networks subject to further topological restrictions have been also widely considered, for instance, planar networks and bipartite networks [6, 19]. Planar graphs are universally used as topologies in the design of printed circuits, such as VLSI circuits [19, 20], while bipartite graphs model several interconnection networks, such as the mesh and the hypercube [9, 19]. In this paper, henceforth we consider bipartite networks.

It is not difficult to see that the degree/diameter problem can be reformulated to consider only graphs of a given class. For instance, the degree/diameter problem for bipartite graphs [15] can be stated as follows.

Degree/diameter problem for bipartite graphs: Given natural numbers $\Delta \geq 2$ and $D \geq 2$, find the largest possible number $N_{\Delta, D}^{b}$ of vertices in a bipartite graph of maximum degree $\Delta$ and diameter $D$.

It is straightforward to verify that $N_{\Delta, D}^{b}$ is well defined for $\Delta \geq 2$ and $D \geq 2$. 
Similar to the general case of the Moore bound, an upper bound for $N_{\Delta, D}^{b}$ is given by the Moore bipartite bound, which is denoted by $M_{\Delta, D}^{b}$ and is defined below.

$$
\begin{aligned}
M_{\Delta, D}^{b} & =1+\Delta+\Delta(\Delta-1)+\cdots+\Delta(\Delta-1)^{D-2}+(\Delta-1)^{D-1} \\
& =2\left(1+(\Delta-1)+\cdots+(\Delta-1)^{D-1}\right) \\
& = \begin{cases}2 \frac{(\Delta-1)^{D}-1}{\Delta-2} & \text { if } \Delta>2 \\
2 D & \text { if } \Delta=2\end{cases}
\end{aligned}
$$

A bipartite graph of maximum degree $\Delta$, diameter $D$ and order $M_{\Delta, D}^{b}$ is called a Moore bipartite graph. Moore bipartite graphs are necessarily regular of degree $\Delta$, and have turned out to be very rare. They exist only when the diameter is 2, 3, 4 or 6 $[15,17]$, and in the cases of $D=3,4$ and 6 , they have been produced only for those values of $\Delta$ such that $\Delta-1$ is a prime power $[3,15]$.

With the exception of $N_{3,5}^{b}=M_{3,5}^{b}-6$, settled in [13], the other known values of $N_{\Delta, D}^{b}$ are those for which there is a Moore bipartite graph.

In this context it is natural to investigate what happens when the order of bipartite graphs misses the Moore bipartite bound by a small number of vertices. So we are prompted to consider bipartite graphs of maximum degree $\Delta$, diameter $D$ and order $M_{\Delta, D}^{b}-\epsilon$, that is, bipartite $(\Delta, D,-\epsilon)$-graphs, where the parameter $\epsilon$ is called the defect.

Conditions for $\epsilon$ under which a bipartite $(\Delta, D,-\epsilon)$-graph must be regular of degree $\Delta$ were established in [8], and one of them is stated below.

Proposition 1.1 [8] For $\epsilon<1+(\Delta-1)+(\Delta-1)^{2}+\cdots+(\Delta-1)^{D-2}, \Delta \geq 3$ and $D \geq 3$, a bipartite $(\Delta, D,-\epsilon)$-graph is regular.

By Proposition 1.1, a bipartite $(\Delta, D,-1)$-graph with $\Delta \geq 3$ and $D \geq 3$ must be regular, implying its two partite sets have the same number of vertices, which is impossible because $M_{\Delta, D}^{b}-1$ is odd. Thus, there is no bipartite $(\Delta, D,-1)$-graph with $\Delta \geq 3$ and $D \geq 3$.

In this paper we analyze the case of defect 2 . Since bipartite $(2, D,-2)$-graphs must be paths of length $2 D-3$, with the exception of $D=3$ they clearly do not exist for $D \geq 2$. In the case of $D=3$ the path of length 3 is the only such graph.

When $\Delta \geq 3$, bipartite $(\Delta, 2,-2)$-graphs need not be regular; they are the complete bipartite graphs with partite sets of orders $\Delta$ and $\Delta-2$. In the following, assume $\Delta \geq 3$ and $D \geq 3$.

Concerning bipartite $(\Delta, D,-2)$-graphs, the paper [8] considered bipartite $(\Delta, 3,-2)$-graphs, deriving some necessary conditions for their existence, and proving the uniqueness of the two known bipartite $(\Delta, 3,-2)$-graphs (both graphs are depicted in Fig. 1). Results about bipartite $(\Delta, D,-2)$-graphs for $\Delta \geq 3$ and $D \geq 4$ were first obtained in [7], where it was proved that the eigenvalues other than $\pm \Delta$ of such graphs are the roots of the polynomials $H_{D-1}(x) \pm 1$, with $H_{D-1}(x)$ being the Dickson polynomial of the second kind with parameter $\Delta-1$ and degree $D-1$ [14]. Moreover, [7] provided some necessary conditions for the existence of bipartite $(\Delta, D,-2)$-graphs, ruled out the existence of bipartite $(\Delta, D,-2)$-graphs for all 


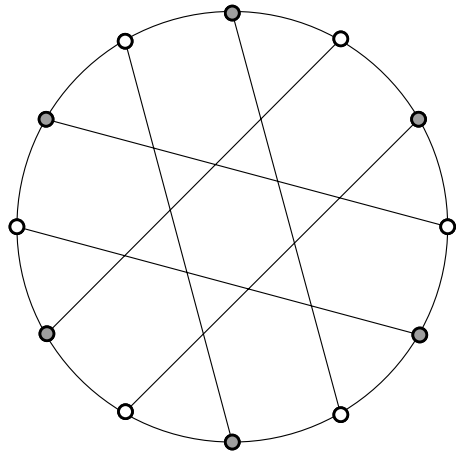

(a)

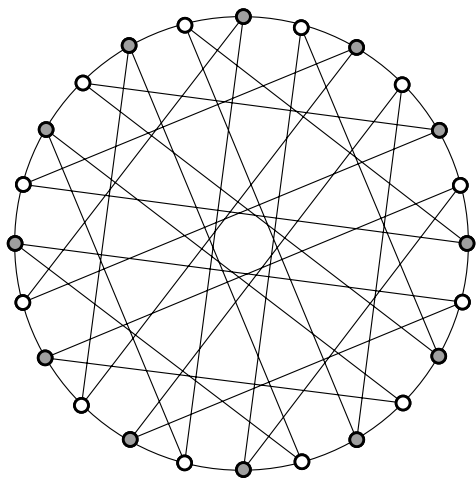

(b)

Fig. 1 Two known bipartite $(\Delta, D,-2)$-graphs for $\Delta \geq 3$ and $D \geq 3$, the unique bipartite $(3,3,-2)$-graph (a) and the unique bipartite $(4,3,-2)$-graph (b)

$\Delta \geq 3$ and $D=4,5,6$ and 8 , and conjectured that bipartite $(\Delta, D,-2)$-graphs for all $\Delta \geq 3$ and all $D \geq 4$ do not exist.

This paper is a follow-up of [7]. Here we prove the aforementioned conjecture by settling the non-existence of bipartite $(\Delta, D,-2)$-graphs for all $\Delta \geq 3$ and all $D \geq 6$. In our proof we are influenced by the reasoning used in the proofs of the non-existence of Moore graphs for $\Delta \geq 3$ and $D \geq 3$ [1], the non-existence of regular graphs of degree $\Delta \geq 3$, even girth $\mathrm{g} \geq 8$ and order $M_{\Delta, \mathrm{g} / 2}^{b}+2$ [5], and the non-existence of regular graphs of degree $\Delta \geq 3$, odd girth $\mathrm{g} \geq 5$ and order $M_{\Delta,(\mathrm{g}-1) / 2}+1$ [2]. We first prove that the multiplicities of the eigenvalues of a hypothetical graph satisfy certain inequalities, and based on these inequalities, we derive that certain sums of two eigenvalues must be integer. But, from another point of view, we can prove that those sums must be in the open interval $(0,1)$, and thus arriving at a contradiction.

As a consequence, for $\Delta \geq 3$ and $D \geq 4$ whenever there exists no Moore bipartite graph, it follows that $N_{\Delta, D}^{b} \leq M_{\Delta, D}^{b}-\overline{4}$.

It is worth acknowledging that some of the computations in the paper were performed with the help of the software Wolfram Mathematica ${ }^{\circledR}$ [18].

\section{Preliminaries}

The terminology used in this paper is standard and consistent with that employed in [4].

The set of edges in a graph $\Gamma$ joining a vertex $x$ in $X \subseteq V(\Gamma)$ to a vertex $y$ in $Y \subseteq V(\Gamma)$ is denoted by $E(X, Y)$.

Let $\Gamma$ be a bipartite graph of diameter $D$, and $u v$ an edge of $\Gamma$. Also, for $0 \leq i \leq$ $D-1$ define the sets $U_{i}$ and $V_{i}$ as follows:

$$
U_{i}=\{z \in V(\Gamma) \mid d(u, z)=i, d(v, z)=i+1\}
$$




$$
V_{i}=\{z \in V(\Gamma) \mid d(v, z)=i, d(u, z)=i+1\}
$$

The decomposition of $\Gamma$ into the sets $U_{i}$ and $V_{i}$ is called the standard decomposition for a graph of even girth with respect to the edge $u v$ [5].

Since $\Gamma$ is bipartite, the sets $U_{i}$ and $V_{i}$ are disjoint for $0 \leq i \leq D-1$.

From now on, $\Gamma$ denotes a bipartite $(\Delta, D,-2)$-graph for $\Delta \geq 3$ and $D \geq 3, n=$ $M_{\Delta, D}^{b}-2$ denotes its order, and $\mathcal{A}$ denotes its adjacency matrix. By Proposition 1.1, $\Gamma$ is regular of degree $\Delta$. Considering the girth of $\Gamma$, denoted $\mathrm{g}(\Gamma)$, it is known that a graph of degree $\Delta$ and girth $2 D$ has order at least $M_{\Delta, D}^{b}$ [4, Proposition 23.1(2)], so $\mathrm{g}(\Gamma) \leq 2 D-2$. But, if $\mathrm{g}(\Gamma) \leq 2 D-4$ then the order of $\Gamma$ would be at most $M_{\Delta, D}^{b}-\overline{2}(\Delta-1)-2$, a contradiction. Therefore, $\mathrm{g}(\Gamma)=2 D-2$.

Proposition 2.1 Every vertex $u$ of $\Gamma$ is contained in exactly one cycle $C_{u}$ of length $2 D-2$.

Proof Consider the standard decomposition for $\Gamma$ with respect to the edge $u v$. Suppose that there are at least two edges joining vertices at $U_{D-2}$ to vertices at $V_{D-2}$, that is, $\left|E\left(U_{D-2}, V_{D-2}\right)\right| \geq 2$. In such a case, $\left|U_{D-1}\right| \leq(\Delta-1)^{D-1}-2$ and $\left|V_{D-1}\right| \leq(\Delta-1)^{D-1}-2$. Therefore,

$$
\begin{aligned}
|V(\Gamma)|= & \sum_{i=0}^{D-1}\left|U_{i}\right|+\sum_{i=0}^{D-1}\left|V_{i}\right| \\
\leq & 2\left(1+(\Delta-1)+(\Delta-1)^{2}+\cdots+(\Delta-1)^{D-2}\right) \\
& +2\left((\Delta-1)^{D-1}-2\right) \\
= & 2\left(1+(\Delta-1)+(\Delta-1)^{2}+\cdots+(\Delta-1)^{D-1}\right)-4 \\
= & M_{\Delta, D}^{b}-4
\end{aligned}
$$

which is a contradiction. Consequently, $0 \leq\left|E\left(U_{D-2}, V_{D-2}\right)\right| \leq 1$.

Suppose $\left|E\left(U_{D-2}, V_{D-2}\right)\right|=1$. Then, since $\left|U_{D-1}\right|=(\Delta-1)^{D-1}-1$ and $\left|V_{D-1}\right|=(\Delta-1)^{D-1}-1$, we obtain our unique cycle $C_{u}$.

Suppose $\left|E\left(X_{D-2}, Y_{D-2}\right)\right|=0$. Then, since $\left|U_{D-1}\right|=(\Delta-1)^{D-1}-1$ and $\left|V_{D-1}\right|=(\Delta-1)^{D-1}-1$, there must exist exactly one vertex $x \in U_{D-1}$ such that $\left|E\left(\{x\}, U_{D-2}\right)\right|=2$, and exactly one vertex $y \in V_{D-1}$ such that $\left|E\left(\{y\}, V_{D-2}\right)\right|=2$. This argument shows the existence of the unique cycle $C_{u}$, which contains $u$ and $x$.

We call the unique vertex on $C_{u}$ at distance $D-1$ from $u$ the repeat of $u$, and we denote it by $\operatorname{rep}(u)$. From now on set $r=\frac{\mathrm{g}(\Gamma)}{2}=D-1$.

Therefore, we have the following lemma.

Lemma 2.1 [7] If a bipartite $(\Delta, D,-2)$-graph exists, then $(2 D-2)$ divides its order $n$.

The fact that rep is an automorphism of $\Gamma$ was proved in [8]. The permutation matrix associated with rep is called the defect matrix of $\Gamma$ and plays an important role in the study of the structure of $\Gamma$ (see [7]). 
In [7] it was proved that the eigenvalues of $\Gamma$, other than $\pm \Delta$, satisfy (1).

Theorem 2.1 [7] If $\theta(\neq \pm \Delta)$ is an eigenvalue of the adjacency matrix $\mathcal{A}$ of $\Gamma$, then

$$
H_{r}(\theta)-\varepsilon=0
$$

where $\varepsilon= \pm 1$.

The polynomials $H_{r}(x)$ satisfy Recurrence Equation (2) [7, 17], and as noted in [7], they are the Dickson polynomials of the second kind with parameter $\Delta-1$ and degree $r$ [14].

$$
\left\{\begin{array}{l}
H_{0}(x)=1 \\
H_{1}(x)=x \\
H_{i+1}(x)=x H_{i}(x)-(\Delta-1) H_{i-1}(x) \text { for } i \geq 1
\end{array}\right.
$$

The roots of $H_{r}(x)$, obtained in [17], are $2 \sqrt{\Delta-1} \cos \frac{i \pi}{r+1}$ for $i=1, \ldots, r$. This result suggests setting $x=-2 \sqrt{\Delta-1} \cos \varphi, 0<\varphi<\pi$, in $H_{r}(x)$, from which we obtain

$$
H_{r}(x)=(-s)^{r} \frac{\sin (r+1) \varphi}{\sin \varphi}, \quad \text { with } s=\sqrt{\Delta-1}
$$

For the rest of the paper let $s=\sqrt{\Delta-1}$.

Now we make the change of variable $\varphi=\frac{i \pi-\alpha}{r+1}$, as suggested in [2, 5]. Then, by using (3), equation (1) transforms into the following function in $\alpha$.

$$
\sin \alpha-\eta_{i} s^{-r} \sin \left(\frac{i \pi-\alpha}{r+1}\right)=0, \quad \text { where } \eta_{i}=\varepsilon(-1)^{r+i+1}
$$

We note that the polynomial $H_{r}(x)$ equals the polynomial $E_{r+1}(x)\left(E_{0}(x)=0\right.$, $E_{1}(x)=1$ and $E_{i+1}(x)=x E_{i}(x)-(\Delta-1) E_{i-1}(x)$ for $\left.i \geq 1\right)$ from [5, p. 4]. Therefore, by substituting $r$ for $r+1$ in Lemma 3.3 from [5], we obtain the following result (the bounds for $\alpha$ are derived from the proof of Lemma 3.3).

Lemma 2.2 (Modification of Lemma 3.3 from [5]) For either value of $\varepsilon$, (1) has $r$ distinct roots $\theta_{1}<\theta_{2}<\cdots<\theta_{r}$, with $\theta_{i}=-2 s \cos \varphi_{i}\left(0<\varphi_{i}<\pi\right)$. Furthermore, if we set $\varphi_{i}=\frac{i \pi-\alpha_{i}}{r+1}$ then

$$
\begin{array}{ll}
0<\alpha_{i}<\min \left\{s^{-r} \varphi_{i}, s^{-r}\left(\pi-\varphi_{i}\right)\right\} & \text { if } \eta_{i}=1 \\
\max \left\{-s^{-r} \varphi_{i},-s^{-r}\left(\pi-\varphi_{i}\right)\right\}<\alpha_{i}<0 & \text { if } \eta_{i}=-1
\end{array}
$$

and consequently

$$
\begin{array}{ll}
\frac{i \pi}{r+1+s^{-r}}<\varphi_{i}<\frac{i \pi}{r+1} & \text { if } \eta_{i}=1 \\
\frac{i \pi}{r+1}<\varphi_{i}<\frac{i \pi}{r+1-s^{-r}} & \text { if } \eta_{i}=-1
\end{array}
$$


By Theorem 2.1 and Lemma 2.2, it follows that the polynomial

$$
\psi(x)=\left(x^{2}-\Delta^{2}\right)\left(H_{r}(x)-1\right)\left(H_{r}(x)+1\right)
$$

has only simple roots and is a multiple of the minimal polynomial of $\Gamma$.

From (2) and (3) we obtain the following assertion.

Proposition 2.2 The roots of the polynomial $\psi(x)=\left(x^{2}-\Delta^{2}\right)\left(H_{r}(x)-1\right)\left(H_{r}(x)+\right.$ 1) are symmetric with respect to 0 , that is, $\theta$ is a root of $\psi(x)$ if, and only if, $-\theta$ is a root of $\psi(x)$.

Proof Suppose $r$ is even. Then, from (2) and (3) it follows that $H_{r}(-x)=H_{r}(x)$, that is, $\theta$ is a root of $H_{r}(x)-\varepsilon$ if, and only if, $-\theta$ is a root of $H_{r}(x)-\varepsilon$, where $\varepsilon= \pm 1$.

Suppose $r$ is odd. Then, from (2) and (3) it follows that $H_{r}(-x)=-H_{r}(x)$, that is, $\theta$ is a root of $H_{r}(x)-\varepsilon$ if, and only if, $-\theta$ is a root of $H_{r}(x)+\varepsilon$, where $\varepsilon= \pm 1$.

\section{Multiplicities of eigenvalues}

In this section we compute the multiplicities of the eigenvalues different from $\pm \Delta$ of $\Gamma$. First some lemmas and some definitions are needed.

Lemma 3.1 (Lemma 3.4 from [10]) Let $\theta$ be a simple root of the polynomial $f(x)$, and put $f_{\theta}(x)=\frac{f(x)}{x-\theta}$. If $M$ is a matrix satisfying $f(M)=0$ then $\frac{\operatorname{tr}\left(f_{\theta}(M)\right)}{f_{\theta}(\theta)}$ is the multiplicity of $\theta$ as an eigenvalue of $M$, and so is rational, where $\operatorname{tr}(M)$ stands for the trace of $M$.

Let $G$ be a Moore bipartite graph of degree $\Delta$ and diameter $D$ (and of girth $2 D$ ), and let $\mathcal{B}_{D}$ be the $(D+1) \times(D+1)$ intersection matrix of $G$. Then, the matrix $\mathcal{B}_{D}$ is defined as follows; see [4, p. 182].

$$
\mathcal{B}_{D}=\left(\begin{array}{cccccccc}
0 & 1 & & & & & & \\
\Delta & 0 & 1 & & & & 0 & \\
& \Delta-1 & 0 & 1 & & & & \\
& & \Delta-1 & 0 & 1 & & & \\
& & & & & \ddots & & \\
& & & & \ddots & \ddots & 1 & \\
& & & & & \Delta-1 & 0 & \Delta \\
& & & & & & \Delta-1 & 0
\end{array}\right)
$$

Let $T_{\Delta}$ denote the infinite $\Delta$-regular tree, and for a graph $G$ and a vertex $u$ of $G$, denote by $N_{G}^{q}(u)$ the number of closed walks of length $q$ starting at $u$. Also, let $(B)_{i, j}$ denote the entry $(i, j)$ in the matrix $B$. 
Lemma 3.2 (Corollary of Proposition 21.2 from [4]) Let $G$ be a Moore bipartite graph of degree $\Delta$ and diameter $D$ (and of girth $2 D$ ). Then, $G$ has $D+1$ distinct eigenvalues, which are the eigenvalues of the matrix $\mathcal{B}_{D}$ of $G$.

Lemma 3.3 [11] In a $\Delta$-regular graph $G$ the number $N_{G}^{q}(u)$ of closed walks of length $q<\mathrm{g}(G)$ starting (and ending) at any vertex $u$ of $G$ equals the number $N_{T_{\Delta}}^{q}(u)$ of such walks starting (and ending) at any vertex $u$ of the infinite $\Delta$-regular tree. Furthermore, the entry $\left(\mathcal{B}_{\left\lceil\frac{\mathrm{g}(G)}{2}\right\rceil}^{q}\right)_{0,0}$ gives this number.

Recall that the number of closed walks of length $q$ in $\Gamma$ is given by $\operatorname{tr}\left(\mathcal{A}^{q}\right)$.

Observation 3.1 Let $u$ be a vertex of $\Gamma$. Since $\mathrm{g}(\Gamma)=2 r$, in $\Gamma$ the number $N_{\Gamma}^{q}(u)$ of closed walks of length $q(q=1, \ldots, 2 r-1)$ starting at $u$ is the same as the number $N_{T_{\Delta}}^{q}(u)$ of closed walks of length $q(q=1, \ldots, 2 r-1)$ starting at $u$ in the infinite $\Delta$-regular tree $T_{\Delta}$ rooted at $u$. By Lemma 3.3, $N_{T_{\Delta}}^{q}(u)=\left(\mathcal{B}_{r+1}^{q}\right)_{0,0}$.

Furthermore, as $\Gamma$ is bipartite, $\Gamma$ contains no closed walk of odd length, assertion that is also true in $T_{\Delta}$.

Thus, it follows that $\operatorname{tr}\left(\mathcal{A}^{q}\right)=n\left(\mathcal{B}_{r+1}^{q}\right)_{0,0}$ for $q=1, \ldots, 2 r-1,2 r+1$.

Theorem 3.1 The multiplicity $\mathrm{m}(\theta)$ of $\theta, \theta \neq \pm \Delta$, as an eigenvalue of $\Gamma$ is

$$
\mathrm{m}(\theta)=\frac{n \Delta(\Delta-1) H_{r-1}(\theta)}{2 H_{r}^{\prime}(\theta)\left(\Delta^{2}-\theta^{2}\right)}-\frac{n \theta}{\varepsilon H_{r}^{\prime}(\theta)\left(\Delta^{2}-\theta^{2}\right)}
$$

where $H_{r}^{\prime}(x)$ is the derivative of $H_{r}(x), \varepsilon= \pm 1$ and $H_{r}(\theta)-\varepsilon=0$.

Proof To compute the multiplicity of an eigenvalue $\theta$ of $\Gamma$, we follow the method suggested in [2]. Consider $\psi(x)=\left(x^{2}-\Delta^{2}\right)\left(H_{r}(x)-1\right)\left(H_{r}(x)+1\right)$ and set $\psi_{\theta}(x)=$ $\frac{\psi(x)}{x-\theta}$. Then, as $\psi(\mathcal{A})=0$, by Lemma $3.1 \mathrm{~m}(\theta)=\frac{\operatorname{tr}\left(\psi_{\theta}(\mathcal{A})\right)}{\psi_{\theta}(\theta)}$.

As $\operatorname{deg}\left(H_{r}(x)\right)=r, \operatorname{deg}\left(\psi_{\theta}(x)\right)=2 r+1$, where $\operatorname{deg}(p(x))$ stands for the degree of the polynomial $p(x)$.

Let us assume that $\psi_{\theta}(x)=x^{2 r+1}+a_{2 r} x^{2 r}+\cdots+a_{1} x+a_{0}$. Then, by virtue of Proposition 2.2 and Viète's formulae, we obtain that $a_{2 r}=\theta$. Furthermore,

$$
\operatorname{tr}\left(\psi_{\theta}(\mathcal{A})\right)=\operatorname{tr}\left(\mathcal{A}^{2 r+1}\right)+a_{2 r} \operatorname{tr}\left(\mathcal{A}^{2 r}\right)+\cdots+a_{1} \operatorname{tr}(\mathcal{A})+a_{0} \operatorname{tr}\left(\mathcal{I}_{n}\right)
$$

Let now $\mathcal{B}_{i+1}$, for $i \geq 0$, be the intersection matrix representing a Moore bipartite graph of girth $2 i+2$ and degree $\Delta$ (in particular, $\mathcal{B}_{1}=\left(\begin{array}{ll}0 & \Delta \\ \Delta & 0\end{array}\right)$ ).

By Observation 3.1, we have $\operatorname{tr}\left(\mathcal{A}^{q}\right)=n\left(\mathcal{B}_{r+1}^{q}\right)_{0,0}$ for $q=1, \ldots, 2 r-1,2 r+1$. Furthermore, since every vertex $u$ of $\Gamma$ is contained in exactly one cycle of length $2 r, N_{\Gamma}^{2 r}(u)=N_{T_{\Delta}}^{2 r}(u)+2$. Thus

$$
\begin{aligned}
\operatorname{tr}\left(\psi_{\theta}(\mathcal{A})\right) & =n\left(\left(\psi_{\theta}\left(\mathcal{B}_{r+1}\right)\right)_{0,0}+2 a_{2 r}\right) \\
& =n\left(\left(\psi_{\theta}\left(\mathcal{B}_{r+1}\right)\right)_{0,0}+2 \theta\right)
\end{aligned}
$$


As $\left(x^{2}-\Delta^{2}\right) H_{r}(x)$ is the minimal polynomial of $\mathcal{B}_{r+1}$ (see [17]), we have

$$
\psi_{\theta}\left(\mathcal{B}_{r+1}\right)=-\frac{\mathcal{B}_{r+1}^{2}-\Delta^{2} \mathcal{I}_{n}}{\mathcal{B}_{r+1}-\theta \mathcal{I}_{n}}
$$

Setting $L_{i+1}(x)=\frac{x^{2}-\Delta^{2}}{x-\theta}\left(H_{i}(x)-H_{i}(\theta)\right)$ for $i=0, \ldots, r$, we get

$$
L_{r+1}\left(\mathcal{B}_{r+1}\right)=-H_{r}(\theta) \frac{\mathcal{B}_{r+1}^{2}-\Delta^{2} \mathcal{I}_{n}}{\mathcal{B}_{r+1}-\theta \mathcal{I}_{n}}=-\varepsilon \frac{\mathcal{B}_{r+1}^{2}-\Delta^{2} \mathcal{I}_{n}}{\mathcal{B}_{r+1}-\theta \mathcal{I}_{n}}
$$

Therefore, $\psi_{\theta}\left(\mathcal{B}_{r+1}\right)=\varepsilon L_{r+1}\left(\mathcal{B}_{r+1}\right)$.

By using $\left((x-\theta) \psi_{\theta}(x)\right)^{\prime}=\left(\left(x^{2}-\Delta^{2}\right)\left(H_{r}^{2}(x)-1\right)\right)^{\prime}$, where the prime ${ }^{\prime}$ indicates the derivative of the corresponding function, we have $\psi_{\theta}(\theta)=2 \varepsilon H_{r}^{\prime}(\theta)\left(\theta^{2}-\Delta^{2}\right)$. Thus

$$
\mathrm{m}(\theta)=\frac{n\left(L_{r+1}\left(\mathcal{B}_{r+1}\right)\right)_{0,0}}{2 H_{r}^{\prime}(\theta)\left(\theta^{2}-\Delta^{2}\right)}+\frac{n \theta}{\varepsilon H_{r}^{\prime}(\theta)\left(\theta^{2}-\Delta^{2}\right)}
$$

We are now interested in finding a recurrence relation for the expression $\left(L_{i+1}\left(\mathcal{B}_{i+1}\right)\right)_{0,0}$. In fact

$$
\begin{aligned}
L_{i+1}(x)= & \frac{x^{2}-\Delta^{2}}{x-\theta}\left(H_{i}(x)-H_{i}(\theta)\right) \\
= & \frac{x^{2}-\Delta^{2}}{x-\theta}\left[x H_{i-1}(x)-(\Delta-1) H_{i-2}(x)\right. \\
& \left.-\left(\theta H_{i-1}(\theta)-(\Delta-1) H_{i-2}(\theta)\right)\right] \\
= & \frac{x^{2}-\Delta^{2}}{x-\theta}\left(x H_{i-1}(x)-\theta H_{i-1}(\theta)\right)-(\Delta-1) L_{i-1}(x) \\
= & \frac{x^{2}-\Delta^{2}}{x-\theta}\left(x H_{i-1}(x)-\theta H_{i-1}(x)\right)+\theta L_{i}(x)-(\Delta-1) L_{i-1}(x) \\
= & \left(x^{2}-\Delta^{2}\right) H_{i-1}(x)+\theta L_{i}(x)-(\Delta-1) L_{i-1}(x)
\end{aligned}
$$

Setting $x=\mathcal{B}_{i}$, we have $L_{i+1}\left(\mathcal{B}_{i}\right)=\theta L_{i}\left(\mathcal{B}_{i}\right)-(\Delta-1) L_{i-1}\left(\mathcal{B}_{i}\right)$.

As the Moore bipartite graphs represented by $\mathcal{B}_{i+1}, \mathcal{B}_{i}, \mathcal{B}_{i-1}$ have girths $2 i+2,2 i$ and $2 i-2$, respectively, $\left(\mathcal{B}_{i+1}^{q}\right)_{0,0}=\left(\mathcal{B}_{i}^{q}\right)_{0,0}=\left(\mathcal{B}_{i-1}^{q}\right)_{0,0}$ for $q=0, \ldots, 2 i-3$ and $i \geq 2$ (this can be deduced by reasoning as in Observation 3.1).

Since $\operatorname{deg}\left(L_{i+1}(x)\right)=i+1,\left(L_{i-1}\left(\mathcal{B}_{i}\right)\right)_{0,0}=\left(L_{i-1}\left(\mathcal{B}_{i-1}\right)\right)_{0,0}$ and $\left(L_{i+1}\left(\mathcal{B}_{i+1}\right)\right)_{0,0}$ $=\left(L_{i+1}\left(\mathcal{B}_{i}\right)\right)_{0,0}$.

Thus, $\left(L_{i+1}\left(\mathcal{B}_{i+1}\right)_{0,0}=\theta\left(L_{i}\left(\mathcal{B}_{i}\right)\right)_{0,0}-(\Delta-1)\left(L_{i-1}\left(\mathcal{B}_{i-1}\right)\right)_{0,0}\right.$, and setting $l_{i+1}=\left(L_{i+1}\left(\mathcal{B}_{i+1}\right)\right)_{0,0}$, we have the desired recurrence relation

$$
\begin{aligned}
l_{0} & =l_{1}=0 \\
l_{2} & =\Delta-\Delta^{2} \\
l_{i+1} & =\theta l_{i}-(\Delta-1) l_{i-1} \quad \text { for } i \geq 2
\end{aligned}
$$


Hence, we obtain that $l_{i+1}=\left(\Delta-\Delta^{2}\right) H_{i-1}(\theta)$ for $i \geq 1$, and the theorem follows, that is,

$$
\mathrm{m}(\theta)=\frac{n \Delta(\Delta-1) H_{r-1}(\theta)}{2 H_{r}^{\prime}(\theta)\left(\Delta^{2}-\theta^{2}\right)}-\frac{n \theta}{\varepsilon H_{r}^{\prime}(\theta)\left(\Delta^{2}-\theta^{2}\right)}
$$

3.1 Multiplicities as functions of $\cos \varphi$

Next we express $\mathrm{m}(\theta)$ with $\theta=-2 s \cos \varphi$ as a function of $\cos \varphi$. But before, we define the following functions $f(z)$ and $g(z)$.

$$
\begin{aligned}
& f(z)=\frac{4 s^{2}\left(1-z^{2}\right)}{\Delta^{2}-4 s^{2} z^{2}} \\
& g(z)=\frac{\Delta(\Delta-1)\left(\sqrt{1-s^{-2 r}\left(1-z^{2}\right)}+s^{-r} z\right)-4 s^{2-r} z}{(r+1) \sqrt{1-s^{-2 r}\left(1-z^{2}\right)}+s^{-r} z}
\end{aligned}
$$

These functions will receive some attention from now on.

Lemma 3.4 For either value of $\varepsilon$, if we set $\theta_{i}=-2 s \cos \varphi_{i}$ for $i=1, \ldots, r$ then

$$
\mathrm{m}\left(\theta_{i}\right)=\frac{n}{4 s^{2}} f\left(\cos \varphi_{i}\right) g\left(\eta_{i} \cos \varphi_{i}\right)
$$

where $f$ and $g$ are defined as above.

Proof By (3), we have

$$
\begin{aligned}
\frac{\mathrm{d} H_{r}(\theta)}{\mathrm{d} \theta} \frac{\mathrm{d} \theta}{\mathrm{d} \varphi} & =\frac{\mathrm{d}}{\mathrm{d} \varphi}\left((-s)^{r} \frac{\sin (r+1) \varphi}{\sin \varphi}\right) \\
& =\frac{(-s)^{r}}{\sin \varphi}((r+1) \cos (r+1) \varphi-\cot \varphi \sin (r+1) \varphi)
\end{aligned}
$$

We now evaluate $\frac{\mathrm{d} H_{r}(\theta)}{\mathrm{d} \theta} \frac{\mathrm{d} \theta}{\mathrm{d} \varphi}$ at $\varphi_{i}=\frac{i \pi-\alpha_{i}}{r+1}$ for $i=1, \ldots, r$.

$$
\begin{aligned}
\frac{\mathrm{d} H_{r}(\theta)}{\mathrm{d} \theta} \frac{\mathrm{d} \theta}{\mathrm{d} \varphi}\left(\varphi_{i}=\frac{i \pi-\alpha_{i}}{r+1}\right) & =\frac{(-s)^{r}}{\sin \varphi_{i}}\left((r+1) \cos \left(i \pi-\alpha_{i}\right)-\cot \varphi_{i} \sin \left(i \pi-\alpha_{i}\right)\right) \\
& =\frac{(-s)^{r}(-1)^{i}}{\sin \varphi_{i}}\left((r+1) \cos \alpha_{i}+\cot \varphi_{i} \sin \alpha_{i}\right)
\end{aligned}
$$

Therefore

$$
H_{r}^{\prime}\left(\theta_{i}\right)=\frac{(-s)^{r}(-1)^{i}}{2 s \sin ^{2} \varphi_{i}}\left((r+1) \cos \alpha_{i}+\cot \varphi_{i} \sin \alpha_{i}\right)
$$

and by (4), we have

$$
H_{r}^{\prime}\left(\theta_{i}\right)=\frac{(-s)^{r}(-1)^{i}}{2 s \sin ^{2} \varphi_{i}}\left((r+1) \cos \alpha_{i}+\eta_{i} s^{-r} \cos \varphi_{i}\right)
$$


Substituting $H_{r-1}\left(\theta_{i}\right)=(-s)^{r-1}(-1)^{i+1} \frac{\sin \left(\varphi_{i}+\alpha_{i}\right)}{\sin \varphi_{i}}$ and $H_{r}^{\prime}\left(\theta_{i}\right)$ in (5), we obtain

$$
\mathrm{m}\left(\theta_{i}\right)=\frac{n \sin \varphi_{i}}{\left(\Delta^{2}-\theta_{i}^{2}\right)} \frac{\Delta(\Delta-1) \sin \left(\varphi_{i}+\alpha_{i}\right)+2 \theta_{i} \eta_{i} s^{1-r} \sin \varphi_{i}}{(r+1) \cos \alpha_{i}+\eta_{i} s^{-r} \cos \varphi_{i}}
$$

Since $\sin \left(\varphi_{i}+\alpha_{i}\right)=\sin \varphi_{i}\left(\cos \alpha_{i}+\eta_{i} s^{-r} \cos \varphi_{i}\right)$ (by (4)), we have

$$
\mathrm{m}\left(\theta_{i}\right)=\frac{n \sin ^{2} \varphi_{i}}{\left(\Delta^{2}-\theta_{i}^{2}\right)} \frac{\Delta(\Delta-1)\left(\cos \alpha_{i}+\eta_{i} s^{-r} \cos \varphi_{i}\right)+2 \theta_{i} \eta_{i} s^{1-r}}{(r+1) \cos \alpha_{i}+\eta_{i} s^{-r} \cos \varphi_{i}}
$$

By (4) and Lemma 2.2, as $\Delta \geq 3$ and $r \geq 2$, it follows that if $\eta_{i}=1$ then $0<$ $\alpha_{i}<\frac{\pi}{2}$ and that if $\eta_{i}=-1$ then $-\frac{\pi}{2}<\alpha_{i}<0$. Therefore, $\cos \alpha_{i}>0$, and $\cos \alpha_{i}=$ $\sqrt{1-s^{-2 r}\left(1-\cos ^{2} \varphi_{i}\right)}$ by (4).

Consequently

$$
\begin{aligned}
\mathrm{m}\left(\theta_{i}\right)= & \frac{n}{4 s^{2}} \frac{4 s^{2}\left(1-\cos ^{2} \varphi_{i}\right)}{\left(\Delta^{2}-4 s^{2} \cos ^{2} \varphi_{i}\right)} \\
& \times \frac{\Delta(\Delta-1)\left(\sqrt{1-s^{-2 r}\left(1-\cos ^{2} \varphi_{i}\right)}+\eta_{i} s^{-r} \cos \varphi_{i}\right)-4 \eta_{i} s^{2-r} \cos \varphi_{i}}{(r+1) \sqrt{1-s^{-2 r}\left(1-\cos ^{2} \varphi_{i}\right)}+\eta_{i} s^{-r} \cos \varphi_{i}}
\end{aligned}
$$

and taking $f(z)=\frac{4 s^{2}\left(1-z^{2}\right)}{\Delta^{2}-4 s^{2} z^{2}}$ (as suggested in $\left.[2,5]\right)$ and

$$
g(z)=\frac{\Delta(\Delta-1)\left(\sqrt{1-s^{-2 r}\left(1-z^{2}\right)}+s^{-r} z\right)-4 s^{2-r} z}{(r+1) \sqrt{1-s^{-2 r}\left(1-z^{2}\right.}+s^{-r} z},
$$

we obtain the desired formula

$$
\mathrm{m}\left(\theta_{i}\right)=\frac{n}{4 s^{2}} f\left(\cos \varphi_{i}\right) g\left(\eta_{i} \cos \varphi_{i}\right)
$$

Corollary 3.1 The polynomial $\psi(x)=\left(x^{2}-\Delta^{2}\right)\left(H_{r}(x)-1\right)\left(H_{r}(x)+1\right)$ is the minimal polynomial of $\Gamma$.

Proof It is not difficult to see that for $|z|<1$, we have $f(z)>0$. To see that $g(z)>0$ for $|z|<1$, we multiply both the numerator and the denominator of $g(z)$ by $s^{r}$. Then,

$$
\begin{aligned}
g(z) & =\frac{\Delta(\Delta-1)\left(s^{r} \sqrt{1-s^{-2 r}\left(1-z^{2}\right)}+z\right)-4 s^{2} z}{s^{r}(r+1) \sqrt{1-s^{-2 r}\left(1-z^{2}\right.}+z} \\
& =\frac{\Delta(\Delta-1) \sqrt{s^{2 r}-1+z^{2}}+\left(\Delta(\Delta-1)-4 s^{2}\right) z}{(r+1) \sqrt{s^{2 r}-1+z^{2}}+z}
\end{aligned}
$$

In the last expression of $g(z)$ we can readily see that, for $|z|<1, g(z)>0$.

Therefore, setting $\theta_{i}=-2 s \cos \varphi_{i}$ with $i=1, \ldots, r$ for either value of $\varepsilon$, it follows that $\mathrm{m}\left(\theta_{i}\right)>0$. Thus, $\theta_{i}$ is an eigenvalue of $\Gamma$. 


\section{Properties of the functions $f$ and $g$}

In the previous section we expressed the multiplicity of an eigenvalue $\theta$ of $\Gamma$ by means of certain functions $f$ and $g$. In this section we obtain several properties and relationships between these functions.

Lemma 4.1 (See Lemma 3.5 from [5]) For $\Delta \geq 3$ and $|z|<1$ the function $f(z)$ is even and concave down.

Lemma 4.2 For $\Delta \geq 3, r \geq 5$ and $|z|<1$, the monotonicity of $g(z)$ behaves as follows.

(i) For $\Delta=3,4$ the function $g(z)$ is monotonic decreasing; and

(ii) for $\Delta \geq 5 \mathrm{~g}(\mathrm{z})$ is monotonic increasing

Proof To prove that $g(z)$ is monotonic increasing (decreasing) for $|z|<1$, it suffices to show that $g^{\prime}(z)$ is positive (negative) along the interval.

$$
g^{\prime}(z)=-\frac{s^{-r}\left(-1+s^{2 r}\right)(\Delta-1)(4(1+r)-r \Delta)}{\sqrt{1+s^{-2 r}\left(-1+z^{2}\right)}\left(z+(1+r) s^{r} \sqrt{1+s^{-2 r}\left(-1+z^{2}\right)}\right)^{2}}
$$

From the expression of $g^{\prime}(z)$, we can verify that $g^{\prime}(z)$ is negative for $\Delta=3,4$ and $r \geq 5$, while it is positive for $\Delta \geq 5$ and $r \geq 5$.

Lemma 4.3 If $\cos \varphi_{2}<-\cos \varphi_{r}$ and $r \geq 5$, the following relationships between the functions $f$ and $g$ hold.

(i) If $\Delta=3,4$ then

$$
\frac{f\left(\cos \varphi_{2}\right)}{f\left(\cos \varphi_{r}\right)}>\frac{g(\cos \pi)}{g(\cos 0)}
$$

with the exception of the pairs $(\Delta, r)=(3,5),(3,6),(3,7)$, and $(3,8) ;$ and (ii) if $\Delta \geq 5$ then

$$
\frac{f\left(\cos \varphi_{2}\right)}{f\left(\cos \varphi_{r}\right)}>\frac{g(\cos 0)}{g(\cos \pi)}
$$

Proof Since $\varphi_{2} \in\left(0, \frac{\pi}{2}\right)$ and $\varphi_{r} \in\left(\frac{\pi}{2}, \pi\right)$ by Lemma 2.2, $\tau=\pi-\varphi_{r} \in\left(0, \frac{\pi}{2}\right)$ and $\tau<\varphi_{2}\left(\right.$ since $\left.\cos \varphi_{2}<-\cos \varphi_{r}=\cos \tau\right)$.

From the expression of $f(\cos \varphi)$, we have

$$
\frac{f\left(\cos \varphi_{2}\right)}{f\left(\cos \varphi_{r}\right)}=\frac{f\left(\cos \varphi_{2}\right)}{f(\cos \tau)}=1+\frac{\cos 2 \tau-\cos 2 \varphi_{2}}{2 \sin ^{2} \tau} \frac{\Delta^{2}-4 s^{2}}{\Delta^{2}-4 s^{2} \cos ^{2} \varphi_{2}}
$$

By the mean value theorem, we have $\frac{\cos 2 \tau-\cos 2 \varphi_{2}}{2 \tau-2 \varphi_{2}}=-\sin 2 \gamma$ for some $\gamma \in\left(\tau, \varphi_{2}\right)$. Consequently

$$
\frac{\cos 2 \tau-\cos 2 \varphi_{2}}{2 \sin ^{2} \tau}=\frac{\left(\varphi_{2}-\tau\right) \sin 2 \gamma}{\sin ^{2} \tau}=\frac{2\left(\varphi_{2}-\tau\right) \sin \gamma \cos \gamma}{\sin ^{2} \tau}
$$


Also, since $0<\tau<\gamma<\varphi_{2}<\frac{\pi}{2}$, we have $\sin \gamma>\sin \tau$ and $\cos \gamma>\cos \varphi_{2}$, and thus

$$
\frac{\cos 2 \tau-\cos 2 \varphi_{2}}{2 \sin ^{2} \tau}>2\left(\varphi_{2}-\tau\right) \frac{\cos \varphi_{2}}{\sin \tau}
$$

By Lemma 2.2, for $i=1, \ldots, r$ if $\eta_{i}=1$ then $\frac{i \pi}{r+1+s^{-r}}<\varphi_{i}<\frac{i \pi}{r+1}$, and $\frac{i \pi}{r+1}<$ $\varphi_{i}<\frac{i \pi}{r+1-s^{-r}}$ otherwise. Therefore, in any case

$$
\frac{i \pi}{r+1+s^{-r}}<\varphi_{i}<\frac{i \pi}{r+1-s^{-r}}
$$

and consequently

$$
\varphi_{2}-\tau=\varphi_{2}+\varphi_{r}-\pi>\frac{2 \pi}{r+1+s^{-r}}+\frac{r \pi}{r+1+s^{-r}}-\pi=\frac{\pi\left(-1+s^{r}\right)}{1+(1+r) s^{r}}
$$

Furthermore, $\cos \varphi_{2}>\cos \frac{2 \pi}{r+1-s^{-r}}>\cos \frac{2 \pi}{5}=\frac{\sqrt{5}-1}{4}>\frac{1}{4}$ (since $r \geq 5$ ), and since $|\sin x| \leq|x|$ for all $x \in \mathbb{R}$, we have

$$
\sin \tau \leq \tau=\pi-\varphi_{r}<\pi-\frac{r \pi}{r+1+s^{-r}}=\frac{\pi\left(1+s^{r}\right)}{1+(1+r) s^{r}}
$$

Therefore

$$
\begin{aligned}
\frac{f\left(\cos \varphi_{2}\right)}{f(\cos \tau)} & >1+\frac{2 \pi\left(-1+s^{r}\right)}{\left(1+(1+r) s^{r}\right)} \frac{1+(1+r) s^{r}}{4 \pi\left(1+s^{r}\right)} \frac{4\left(\Delta^{2}-4 s^{2}\right)}{4 \Delta^{2}-s^{2}} \\
& =1+\frac{2\left(-1+s^{r}\right)}{1+s^{r}} \frac{\Delta^{2}-4 s^{2}}{4 \Delta^{2}-s^{2}}
\end{aligned}
$$

Proof of Claim (i) From the expression of $g(z)$, we obtain

$$
\frac{g(-1)}{g(1)}=1+\frac{2 s^{r}(4(r+1)-r \Delta)}{\left(-1+s^{r}(r+1)\right)\left(\Delta\left(1+s^{r}\right)-4\right)}
$$

Therefore, considering (7) and (8), it suffices to show that, for $\Delta=3,4$ and $r \geq 5$, with the exceptions of the pairs $(\Delta, r)=(3,5),(3,6),(3,7)$, and $(3,8)$, the following inequality holds:

$$
\frac{-1+s^{r}}{1+s^{r}} \frac{\Delta^{2}-4 s^{2}}{4 \Delta^{2}-s^{2}}>\frac{s^{r}(4(r+1)-r \Delta)}{\left(-1+s^{r}(r+1)\right)\left(\Delta\left(1+s^{r}\right)-4\right)}
$$

or equivalently that

$$
h(r)=\frac{-1+s^{r}}{1+s^{r}} \frac{\Delta^{2}-4 s^{2}}{4 \Delta^{2}-s^{2}}-\frac{s^{r}(4(r+1)-r \Delta)}{\left(-1+s^{r}(r+1)\right)\left(\Delta\left(1+s^{r}\right)-4\right)}>0
$$

Indeed, using $s=\sqrt{\Delta-1}$, for $\Delta=3$ and $r \geq 9$ we have

$$
h(r)=\frac{-1-(131+33 r) 2^{\frac{r}{2}}-143 \cdot 2^{r}-19 r 2^{1+r}+(3+3 r) 2^{3 \frac{r}{2}}}{34\left(1+2^{r / 2}\right)\left(-1+3 \cdot 2^{\frac{r}{2}}\right)\left(-1+(1+r) 2^{\frac{r}{2}}\right)}>0
$$


(As the numerator of $h(r)$ is clearly monotonic increasing, with value $-248321+$ $238912 \sqrt{2}>0$ at $r=9$.) While for $\Delta=4$ and $r \geq 4$ we have

$$
h(r)=\frac{-57-4 r 3^{\frac{r}{2}}-23 \cdot 3^{1+\frac{r}{2}}+(4+4 r) 3^{r}}{61\left(1+3^{r / 2}\right)\left(-1+(1+r) 3^{\frac{r}{2}}\right)}>0
$$

(As the numerator of $h(r)$ is clearly monotonic increasing, with value 798 at $r=4$.)

Thus we obtain the claim.

Proof of Claim (ii) It is proved analogously to Claim (i). A complete proof of this claim can be found in [16, p. 112].

This completes the proof of the lemma.

\section{Main result}

In this section we prove the main result of this paper (Theorem 5.1).

Theorem 5.1 Bipartite ( $\Delta, D,-2)$-graphs for $\Delta \geq 3$ and $D \geq 6$ do not exist.

To prove the theorem we prepare two more lemmas, and some definitions.

Henceforth, let $\lambda_{1}<\lambda_{2}<\cdots<\lambda_{r}$ be the roots of $H_{r}(x)-1=0$, and let $\rho_{1}<$ $\rho_{2}<\cdots<\rho_{r}$ be the roots of $H_{r}(x)+1=0$.

Lemma 5.1 Let $\lambda_{1}, \ldots, \lambda_{r}$ and $\rho_{1}, \ldots, \rho_{r}$ be defined as above. Then the following assertions hold for $\Delta \geq 3$ and $r \geq 5$.

(i) If $r$ is even then $\mathrm{m}\left(\lambda_{i}\right)=\mathrm{m}\left(\lambda_{1+r-i}\right)$ and $\mathrm{m}\left(\rho_{i}\right)=\mathrm{m}\left(\rho_{1+r-i}\right)$, whereas if $r$ is odd then $\mathrm{m}\left(\lambda_{i}\right)=\mathrm{m}\left(\rho_{1+r-i}\right)$, for $1 \leq i \leq r$; and

(ii) If $r$ is even then $\mathrm{m}\left(\rho_{1}\right)<\mathrm{m}\left(\rho_{i}\right)$, whereas if $r$ is odd then $\mathrm{m}\left(\lambda_{1}\right)<\mathrm{m}\left(\lambda_{i}\right)$, for $i=2, \ldots, r-1$, and any pair $(\Delta, r) \neq(3,5),(3,6),(3,7),(3,8) ;$ and

(iii) $\mathrm{m}\left(\lambda_{r}\right)<\mathrm{m}\left(\lambda_{i}\right)$ for $i=2, \ldots, r-1$

Proof Next we prove each claim in order.

Proof of Claim (i) If $r$ is even, it follows that $H_{r}(-x)=H_{r}(x)$ (see (3)) and that $\lambda_{i}+\lambda_{1+r-i}=\rho_{i}+\rho_{1+r-i}=0$, and thus, by checking (5), $\mathrm{m}\left(\lambda_{i}\right)=\mathrm{m}\left(\lambda_{1+r-i}\right)$ and $\mathrm{m}\left(\rho_{i}\right)=\mathrm{m}\left(\rho_{1+r-i}\right)$ for $1 \leq i \leq r$. If instead $r$ is odd, $H_{r}(-x)=-H_{r}(x)$; therefore, $\lambda_{i}+\rho_{1+r-i}=0$ and $\mathrm{m}\left(\lambda_{i}\right)=\mathrm{m}\left(\rho_{1+r-i}\right)$ for $1 \leq i \leq r$.

Proof of Claim (ii) In the proof of this claim we consider the trigonometric form of the multiplicity of an eigenvalue of $\Gamma$ (Lemma 3.4).

Assume $r$ is even, $\varepsilon=-1$, and $\rho_{i}=-2 s \cos \varphi_{i}$ for $i=1, \ldots, r$. Then $\eta_{i}=(-1)^{i}$.

By $(i),-\cos \varphi_{1}=\cos \varphi_{r}$. Then, since $-\cos \varphi_{1}=\cos \varphi_{r}<\cos \varphi_{i}<\cos \varphi_{1}$ (for $i \in\{2, \ldots, r-1\})$, by Lemma 4.1 we have

$$
f\left(\cos \varphi_{1}\right)<f\left(\cos \varphi_{i}\right) \quad \text { for } i=2, \ldots, r-1
$$


First suppose $\Delta \geq 5$ and $r \geq 5$. Since $\cos \varphi_{i}<\left|\cos \varphi_{1}\right|$, we obtain that $g\left(-\cos \varphi_{1}\right)$ $<g\left( \pm \cos \varphi_{i}\right)$ for $i=2, \ldots, r-1$ (by Lemma 4.2(ii)), and thus, $\mathrm{m}\left(\rho_{1}\right)<\mathrm{m}\left(\rho_{i}\right)$.

Suppose $\Delta=3,4$ and $r \geq 5$. In this case $g$ is monotonic decreasing (by Lemma 4.2(i)), so we cannot use the same argument as before. Here we also want to prove

$$
f\left(\cos \varphi_{1}\right) g\left(-\cos \varphi_{1}\right)<f\left(\cos \varphi_{i}\right) g\left(\eta_{i} \cos \varphi_{i}\right) \quad \text { for } i=2, \ldots, r-1
$$

Since $\cos 0>\cos \varphi_{i}>\cos \pi$, we have $g(\cos 0)<g\left(\cos \varphi_{i}\right)<g(\cos \pi)$. Then

$$
\begin{aligned}
f\left(\cos \varphi_{1}\right) g\left(-\cos \varphi_{1}\right) & <f\left(\cos \varphi_{1}\right) g(\cos \pi) \text { and } f\left(\cos \varphi_{i}\right) g(\cos 0) \\
& <f\left(\cos \varphi_{i}\right) g\left(\eta_{i} \cos \varphi_{i}\right)
\end{aligned}
$$

Consequently, it suffices to prove that, for any pair $(\Delta, r)$ other than $(3,5),(3,6)$, $(3,7),(3,8)$,

$$
\frac{f\left(\cos \varphi_{i}\right)}{f\left(\cos \varphi_{1}\right)}>\frac{g(\cos \pi)}{g(\cos 0)}
$$

Besides, since $f\left(\cos \varphi_{2}\right) \leq f\left(\cos \varphi_{i}\right)$ for $i=2, \ldots, r-1$, we can equivalently prove that

$$
\frac{f\left(\cos \varphi_{2}\right)}{f\left(\cos \varphi_{r}\right)}>\frac{g(\cos \pi)}{g(\cos 0)}
$$

and as $\cos \varphi_{2}<\cos \varphi_{1}=-\cos \varphi_{r}$, such an inequality follows from Lemma 4.3(i). Therefore, $\mathrm{m}\left(\rho_{1}\right)<\mathrm{m}\left(\rho_{i}\right)$.

Now assume that $r$ is odd, $\varepsilon=1, \lambda_{i}=-2 s \cos \varphi_{i}$, and $\rho_{i}=-2 s \cos \sigma_{i}$ for $i=$ $1, \ldots, r$. Then $\eta_{i}=(-1)^{i}$.

Since $\lambda_{1}<\lambda_{i}, \eta_{i} \cos \varphi_{i}>-\cos \varphi_{1}$ for $\eta_{i}=-1$. Moreover, since $r$ is odd, we obtain that $\rho_{i}+\lambda_{1+r-i}=0$ by virtue of $(i)$. We next prove that $\eta_{i} \cos \varphi_{i}>-\cos \varphi_{1}$ for $\eta_{i}=1$. Since $\cos \varphi_{1}>0$ by Lemma 2.2, we only consider the case of $\cos \varphi_{i}<0$. By Lemma 2.2

$$
\frac{i \pi}{r+1+s^{-r}}<\varphi_{i}<\frac{i \pi}{r+1} \text { and } \quad \frac{i \pi}{r+1}<\sigma_{i}<\frac{i \pi}{r+1-s^{-r}}
$$

As a consequence, $\varphi_{i}<\sigma_{i}$, and since $\varphi_{i}, \sigma_{i} \in(0, \pi)$, it follows that $\cos \varphi_{i}>\cos \sigma_{i}$. Since $\rho_{r}=2 s \cos \varphi_{1} \geq \rho_{i}=-2 s \cos \sigma_{i}>-2 s \cos \varphi_{i}, \cos \varphi_{i}>-\cos \varphi_{1}$. That is

$$
-\cos \varphi_{1}<\cos \varphi_{i}<\cos \varphi_{1} \quad \text { for } i=2, \ldots, r
$$

Then, by Lemma 4.1, $f\left(\cos \varphi_{1}\right)<f\left(\cos \varphi_{i}\right)$ for $i=2, \ldots, r$.

First suppose $\Delta \geq 5$ and $r \geq 5$. By Lemma 4.2(ii), $g\left(-\cos \varphi_{1}\right)<g\left( \pm \cos \varphi_{i}\right)$ for $i=2, \ldots, r-1$, and thus, $\mathrm{m}\left(\lambda_{1}\right)<\mathrm{m}\left(\lambda_{i}\right)$.

Suppose $\Delta=3,4$ and $r \geq 5$. Since $\cos 0>\cos \varphi_{i}>\cos \pi$, by Lemma 4.2(i) we have $g(\cos 0)<g\left(\cos \varphi_{i}\right)<g(\cos \pi)$. Therefore, as above, we only need to show that

$$
\frac{f\left(\cos \varphi_{i}\right)}{f\left(\cos \varphi_{1}\right)}>\frac{g(\cos \pi)}{g(\cos 0)}
$$


or alternatively, since $f\left(\cos \varphi_{1}\right)<f\left(\cos \varphi_{r}\right)$ and $f\left(\cos \varphi_{2}\right) \leq f\left(\cos \varphi_{i}\right)$ for $i=$ $2, \ldots, r-1$, that

$$
\frac{f\left(\cos \varphi_{2}\right)}{f\left(\cos \varphi_{r}\right)}>\frac{g(\cos \pi)}{g(\cos 0)}
$$

Indeed, by (i) we have $-\cos \varphi_{r}=\cos \sigma_{1}$, and by Lemma 2.2, we have $\varphi_{2}>\sigma_{1}$, which implies that $\cos \varphi_{2}<\cos \sigma_{1}$. Consequently, the inequality $\frac{f\left(\cos \varphi_{2}\right)}{f\left(\cos \varphi_{r}\right)}>\frac{g(\cos \pi)}{g(\cos 0)}$ follows from Lemma 4.3(i), and thus, $\mathrm{m}\left(\lambda_{1}\right)<\mathrm{m}\left(\lambda_{i}\right)$.

Proof of Claim (iii) It is proved similarly to Claim (ii) using Lemma 4.3(ii). A complete proof of this claim can be found in [16, p. 119].

This completes the proof of the lemma.

Next we rule out the pairs $(\Delta, r)$ not covered in the previous lemma, that is, $(3,5)$, $(3,6),(3,7)$ and $(3,8)$.

Lemma 5.2 There are no bipartite $(3, D,-2)$-graphs for $D=6,7,8,9$.

Proof The non-existence of bipartite $(3, D,-2)$-graphs with $D=6,8,9$ follows from Lemma 2.1. Indeed, the condition $2(D-1) \mid n$ implies for cubic graphs that $(D-1) \mid\left(2^{D}-2\right)$, and the values $6,8,9$ do not pass the test. To prove the nonexistence of bipartite $(3,7,-2)$-graphs, we use the fact that the multiplicity of each eigenvalue of the hypothetical graph must be integer. Set $\varepsilon=1$. For $\Delta=3$, $H_{6}(\theta)=-8+24 \theta^{2}-10 \theta^{4}+\theta^{6}, H_{6}(\theta)-1=\left(-3+\theta^{2}\right)\left(3-7 \theta^{2}+\theta^{4}\right), H_{6}^{\prime}(\theta)=$ $48 \theta-40 \theta^{3}+6 \theta^{5}$, and $H_{5}(\theta)=\theta\left(12-8 \theta^{2}+\theta^{4}\right)$. The order of such a hypothetical graph is 252. By Theorem 3.1

$$
\mathrm{m}(\theta)=-\frac{252 \theta}{\left(9-\theta^{2}\right)\left(48 \theta-40 \theta^{3}+6 \theta^{5}\right)}+\frac{756\left(12 \theta-8 \theta^{3}+\theta^{5}\right)}{\left(9-\theta^{2}\right)\left(48 \theta-40 \theta^{3}+6 \theta^{5}\right)}
$$

Considering the eigenvalue $\theta=\sqrt{3}$, we have $\mathrm{m}(\sqrt{3})=\frac{70}{3}$, a contradiction. Thus, there are no bipartite $(3,7,-2)$-graphs, and the lemma follows.

Theorem 5.1 Bipartite $(\Delta, D,-2)$-graphs for $\Delta \geq 3$ and $D \geq 6$ do not exist.

Proof of Theorem 5.1 A proof of the non-existence of bipartite $(\Delta, D,-2)$-graphs for all $\Delta \geq 3$ and $D=4,5$ was obtained in [7], so we can assume $D=r+1 \geq 6$. By Lemma 5.2, we can additionally assume that for $\Delta \geq 3$ and $r \geq 5$, the pair $(\Delta, r)$ is different from $(3,5),(3,6),(3,7)$ or $(3,8)$.

Suppose $r$ is odd. By Lemma 5.1(ii) and (iii), we have $\mathrm{m}\left(\lambda_{1}\right) \neq \mathrm{m}\left(\lambda_{i}\right)$ and $\mathrm{m}\left(\lambda_{r}\right) \neq \mathrm{m}\left(\lambda_{i}\right)$ for $i=2, \ldots, r-1$. Therefore, $\lambda_{1}$ and $\lambda_{r}$ are either conjugate quadratic irrationals or integers, ${ }^{1}$ and thus, $\lambda_{1}+\lambda_{r} \in \mathbb{Z}$.

\footnotetext{
${ }^{1}$ Recall that, if $\alpha_{i}$ and $\alpha_{j}$ are eigenvalues of a real square matrix $A$ with rational entries, such that $\alpha_{i}$ and $\alpha_{j}$ are algebraic conjugates over $\mathbb{Q}$, then $\mathrm{m}\left(\alpha_{i}\right)=\mathrm{m}\left(\alpha_{j}\right)$. 
By Lemma 2.2, $\lambda_{1}=-2 s \cos \varphi_{1}$ and $\lambda_{r}=-2 s \cos \varphi_{r}$, where

$$
\begin{aligned}
& \frac{\pi}{r+1}<\varphi_{1}<\frac{\pi}{r+1-s^{-r}} \\
& \frac{r \pi}{r+1}<\varphi_{r}<\frac{r \pi}{r+1-s^{-r}}
\end{aligned}
$$

We have $\lambda_{1}+\lambda_{r}=-2 s\left(\cos \varphi_{1}+\cos \varphi_{r}\right)$. By (9), $\lambda_{1}+\lambda_{r}>-2 s\left(\cos \frac{\pi}{r+1}+\right.$ $\left.\cos \frac{r \pi}{r+1}\right)=0$, because $\pi-\frac{\pi}{r+1}=\frac{r \pi}{r+1}$.

By Lemma 5.1(i), $\lambda_{r}=-\rho_{1}<2 s \cos \frac{\pi}{r+1+s^{-r}}$. Therefore

$$
\begin{aligned}
\lambda_{1}+\lambda_{r}< & 2 s\left(-\cos \frac{\pi}{r+1-s^{-r}}+\cos \frac{\pi}{r+1+s^{-r}}\right) \\
= & 2 s\left(-2 \sin \frac{1}{2}\left(\frac{\pi}{r+1+s^{-r}}-\frac{\pi}{r+1-s^{-r}}\right)\right. \\
& \left.\times \sin \frac{1}{2}\left(\frac{\pi}{r+1+s^{-r}}+\frac{\pi}{r+1-s^{-r}}\right)\right) \\
< & 4 s \frac{1}{2}\left(\frac{\pi}{r+1-s^{-r}}-\frac{\pi}{r+1+s^{-r}}\right) \frac{1}{2}\left(\frac{\pi}{r+1+s^{-r}}+\frac{\pi}{r+1-s^{-r}}\right) \\
= & s\left[\left(\frac{\pi}{r+1-s^{-r}}\right)^{2}-\left(\frac{\pi}{r+1+s^{-r}}\right)\right]=4 \frac{\pi^{2}(r+1) s^{1-r}}{\left((r+1)^{2}-s^{-2 r}\right)^{2}} \\
< & 4 \frac{\pi^{2}(r+1) s^{1-r}}{\left((r+1)^{2}-(r+1)\right)^{2}}=4 \frac{\pi^{2}(r+1) s^{1-r}}{r^{2}(r+1)^{2}}=4 \frac{\pi^{2} s^{1-r}}{r^{2}(r+1)}<1
\end{aligned}
$$

Thus, $0<\lambda_{1}+\lambda_{r}<1$, a contradiction.

If instead $r$ is even, by Lemma 5.1, we have $\rho_{1}$ and $\rho_{r}=-\rho_{1}$ are either conjugate quadratic irrationals or integers. Therefore, $\rho_{1}^{2} \in \mathbb{Z}$. Analogously, we have $\lambda_{r}^{2}=$ $\lambda_{1}^{2} \in \mathbb{Z}$. Hence, $\lambda_{1}^{2}-\rho_{1}^{2} \in \mathbb{Z}$.

By Lemma 2.2

$$
\begin{aligned}
-2 s \cos \frac{\pi}{r+1+s^{-r}}<\lambda_{1} & <-2 s \cos \frac{\pi}{r+1} \\
-2 s \cos \frac{\pi}{r+1} & <\rho_{1}<-2 s \cos \frac{\pi}{r+1-s^{-r}}
\end{aligned}
$$

Then, as $\lambda_{1}^{2}>4 s^{2} \cos ^{2} \frac{\pi}{r+1}$ and $\rho_{1}^{2}<4 s^{2} \cos ^{2} \frac{\pi}{r+1}$, we have $\lambda_{1}^{2}-\rho_{1}^{2}>0$.

Furthermore, as $\lambda_{1}^{2}<4 s^{2} \cos ^{2} \frac{\pi}{r+1+s^{-r}}$ and $\rho_{1}^{2}>4 s^{2} \cos ^{2} \frac{\pi}{r+1-s^{-r}}$, we have

$$
\begin{aligned}
\lambda_{1}^{2}-\rho_{1}^{2} & <4 s^{2}\left(\cos ^{2} \frac{\pi}{r+1+s^{-r}}-\cos ^{2} \frac{\pi}{r+1-s^{-r}}\right) \\
& =4 s^{2}\left(\sin ^{2} \frac{\pi}{r+1-s^{-r}}-\sin ^{2} \frac{\pi}{r+1+s^{-r}}\right)
\end{aligned}
$$




$$
\begin{aligned}
= & 4 s^{2}\left(\sin \frac{\pi}{r+1-s^{-r}}-\sin \frac{\pi}{r+1+s^{-r}}\right) \\
& \times\left(\sin \frac{\pi}{r+1-s^{-r}}+\sin \frac{\pi}{r+1+s^{-r}}\right) \\
= & 16 s^{2} \sin \frac{\pi s^{-r}}{(r+1)^{2}-s^{-2 r}} \cos \frac{\pi(r+1)}{(r+1)^{2}-s^{-2 r}} \\
& \times \sin \frac{\pi(r+1)}{(r+1)^{2}-s^{-2 r}} \cos \frac{\pi s^{-r}}{(r+1)^{2}-s^{-2 r}} \\
< & 16 \frac{\pi^{2} s^{-r+2}(r+1)}{\left((r+1)^{2}-s^{-2 r}\right)^{2}} \cos \frac{\pi(r+1)}{(r+1)^{2}-s^{-2 r} \cos \frac{\pi s^{-r}}{(r+1)^{2}-s^{-2 r}}} \\
& \quad(\operatorname{as}|\sin x| \leq|x| \forall x \in \mathbb{R}) \\
< & 16 \frac{\pi^{2} s^{-r+2}(r+1)}{\left((r+1)^{2}-s^{-2 r}\right)^{2}}<16 \frac{\pi^{2} s^{-r+2}(r+1)}{\left((r+1)^{2}-(r+1)\right)^{2}}=16 \frac{\pi^{2} s^{-r+2}}{r^{2}(r+1)}<1
\end{aligned}
$$

Thus, $0<\lambda_{1}^{2}-\rho_{1}^{2}<1$, a contradiction, and the theorem follows.

\section{Conclusions}

In this paper we have proved the non-existence of bipartite $(\Delta, D,-2)$-graphs for all $\Delta \geq 3$ and all $D \geq 6$, result that, combined with [7], assert that there are no bipartite $(\Delta, D,-2)$-graphs for all $\Delta \geq 3$ and all $D \geq 4$. Consequently, the existence or otherwise of bipartite $(\Delta, D,-2)$-graphs is open only for $D=3$.

Interestingly enough, for a given $\Delta_{0}$ if the defect $\epsilon_{0}=f\left(\Delta_{0}\right)\left(\epsilon_{0}\right.$ is a function of $\Delta_{0}$, and thus, independent of $D$ ) then there exists a constant $D_{0}$ such that a hypothetical (or real) bipartite $\left(\Delta_{0}, D,-\epsilon_{0}\right)$-graph for $D \geq D_{0}$ must be regular. To see this, consider a bipartite graph $\Lambda$ of maximum degree $\Delta_{0}$ and diameter $D_{0}$ with a vertex $u$ of degree at most $\Delta_{0}-1$, and an edge $u v$ of $\Lambda$. Then we use the standard decomposition for a bipartite graph with respect to an edge $u v$ [5]. For $0 \leq i \leq D_{0}-1$ we count the vertices at distance $i$ from $u$ and at distance $i+1$ from $v$, and the vertices at distance $i$ from $v$ and at distance $i+1$ from $u$. Then we count at most $T_{0}^{b}\left(\Delta_{0}, D_{0}\right)=M_{\Delta_{0}, D_{0}}^{b}-\left(1+\left(\Delta_{0}-1\right)+\cdots+\left(\Delta_{0}-1\right)^{D_{0}-2}\right)$ vertices in $\Lambda$.

Therefore, taking $D_{0}$ as the least number such that $M_{\Delta_{0}, D_{0}}^{b}-\epsilon_{0}>T_{0}^{b}\left(\Delta_{0}, D_{0}\right)$, we obtain that any bipartite $\left(\Delta_{0}, D,-\epsilon_{0}\right)$-graph with $D \geq D_{0}$ must be regular, and thus, $\epsilon_{0}$ must be even. Therefore, we have proved

Proposition 6.1 For a given $\Delta_{0}$ and $\epsilon_{0}=f\left(\Delta_{0}\right)$ there exists a constant $D_{0}$ such that any bipartite $\left(\Delta_{0}, D,-\epsilon_{0}\right)$-graph for $D \geq D_{0}$ must be regular, and consequently, $\epsilon_{0}$ must be even.

Then the next interesting case occurs when $\epsilon=4$. In this case each vertex of $\Gamma$ has two repeats, and consequently, the defect matrix can be considered as a direct sum of circulant matrices. Therefore, the spectrum of the defect matrix is not specified as 
in the case of defect 2, thereby making it difficult to apply the same approach to this case.

In general we believe that the following conjecture holds. However, we feel that to make a breakthrough in its proof or disproof, new techniques are required.

Conjecture 6.1 For a given $\Delta_{0}$ and $\epsilon_{0}=f\left(\Delta_{0}\right)$ there exists a constant $D_{1} \geq D_{0}$ such that regular bipartite $\left(\Delta_{0}, D,-\epsilon_{0}\right)$-graphs with $D \geq D_{1}$ do not exist.

\section{Contributions to the degree/diameter problem for bipartite graphs}

For those combinations of $\Delta \geq 3$ and $D \geq 4$ where the non-existence of Moore bipartite graphs of degree $\Delta$ and diameter $D$ is known, we have showed that

$$
N_{\Delta, D}^{b} \leq M_{\Delta, D}^{b}-4
$$

Acknowledgements The author would like to thank Professors Charles Delorme, Leif K. Jørgensen and Mirka Miller, and Dr. Hebert Pérez-Rosés for valuable discussions in the course of this research, and for checking earlier versions of the paper.

The author would also like to thank the referee for many helpful suggestions, which, with no doubt, have improved the presentation of the paper.

\section{References}

1. Bannai, E., Ito, T.: On finite Moore graphs. J. Math. Sci., Univ. Tokyo 20, 191-208 (1973)

2. Bannai, E., Ito, T.: Regular graphs with excess one. Discrete Math. 37, 147-158 (1981). doi:10.1016/0012-365X(81)90215-6

3. Benson, C.T.: Minimal regular graphs of girth eight and twelve. Can. J. Math. 18, 1091-1094 (1966)

4. Biggs, N.L.: Algebraic Graph Theory, 2nd edn. Cambridge University Press, Cambridge (1993)

5. Biggs, N.L., Ito, T.: Graphs with even girth and small excess. Math. Proc. Camb. Philos. Soc. 88(1), 1-10 (1980)

6. Dally, W.J., Towles, B.P.: Principles and Practices of Interconnection Networks. Morgan Kaufmann, San Francisco (2004)

7. Delorme, C., Jørgensen, L.K., Miller, M., Pineda-Villavicencio, G.: On bipartite graphs of defect 2. Eur. J. Comb. 30(4), 798-808 (2009)

8. Delorme, C., Jørgensen, L.K., Miller, M., Pineda-Villavicencio, G.: On bipartite graphs of diameter 3 and defect 2. J. Graph Theory 61(4), 271-288 (2009)

9. Duato, J., Yalamanchili, S., Ni, L.: Interconnection Networks: An Engineering Approach. Morgan Kaufmann, San Francisco (2003). Revised printing ed.

10. Feit, W., Higman, G.: The nonexistence of certain generalized polygons. J. Algebra 1, 114-131 (1964). doi:10.1016/0021-8693(64)90028-6

11. Godsil, C.D., McKay, B.D.: Feasibility conditions for the existence of walk-regular graphs. Linear Algebra Appl. 30, 51-61 (1980)

12. Heydemann, M.C.: Cayley graphs and interconnection networks. In: Graph Symmetry: Algebraic Methods and Applications. NATO ASI Series, Series C: Mathematical and Physical Sciences, vol. 497. Kluwer Academic, Dordrecht (1996)

13. Jørgensen, L.K.: Nonexistence of certain cubic graphs with small diameters. Discrete Math. 114, 265-273 (1993). doi:10.1016/0012-365X(93)90371-Y

14. Lidl, R., Mullen, G.L., Turnwald, G.: Dickson Polynomials. Pitman Monographs and Surveys in Pure and Applied Mathematics, vol. 65. Logman, London (1993)

15. Miller, M., Širáň, J.: Moore graphs and beyond: A survey of the degree/diameter problem. Electron. J. Comb. 1-61 (2005). Dynamic survey DS14

16. Pineda-Villavicencio, G.: Topology of interconnection networks with given degree and diameter. PhD Thesis, School of Information Technology and Mathematical Sciences, University of Ballarat, Ballarat, Australia, Dec 2009. http://guillermo.com.au/pdfs/GPVPhDThesis.pdf 
17. Singleton, R.C.: On minimal graphs of maximum even girth. J. Comb. Theory 1(3), 306-332 (1966). doi:10.1016/S0021-9800(66)80054-6

18. Wolfram, S.: The Mathematica Book, 4th edn. Cambridge University Press, London (1999)

19. Xu, J.: Topological Structure and Analysis of Interconnection Networks. Network Theory and Applications, vol. 7. Kluwer Academic, Dordrecht (2001)

20. Xu, J.: Theory and Application of Graphs. Network Theory and Applications, vol. 10. Kluwer Academic, Dordrecht (2003) 